\title{
Content Analysis of Journal of Aging and Health, 2005-2014
}

\author{
Xianghong Zhao ${ }^{1, a}$, David Royse ${ }^{2, b}$ \\ ${ }^{1}$ School of Law, Jiangnan University, Wuxi, Jiangsu, CHINA; \\ ${ }^{2}$ College of Social Work, University of Kentucky, Lexington, USA. \\ a150115778@qq.com, bdroyse@uky.edu
}

\begin{abstract}
Keywords: Aging research, content analysis, Journal of Aging and Health, gerontology.
\end{abstract}
\begin{abstract}
Objective: The purpose of this study was to analyse the content of Journal of Aging and Health to identify prevalent topics, trends, and under-represented areas as suggested by the keywords associated with articles published from 2005 through 2014. Methods: Keywords associated with each article were entered into an Excel database. After reviewing and discussing the lists of keywords multiple times, the investigators created 23 topical categories. Results: There were 1324 unique keywords and 2572 total. The five largest keyword categories were Aged/Geriatric, Study, Physical disability, Health, and Demographic. The smallest of the categories, "Sex," contained eight keywords. Caregiver/caregiving appeared 31 times. Overall, the keywords "Hispanic" "Latino" and "Mexican American" outnumbered those for African Americans. Discussion: For several topics, keywords appeared infrequently and suggest areas where more work may be needed. Keywords appearing only once in 10 years were: hospice, program evaluation, cost-benefit, suicide, and assistive technology.
\end{abstract}

\section{Introduction}

Topics of interest to professional journals have a certain ebb and flow. Current events, policy changes, and research developments can stimulate new scholarship. While journal editors can sometimes stimulate or highlight certain areas by calling for papers and creating special issues, changes over a decade may be so subtle and slight that they might not be observed by someone in the field.

Content analysis, a technique proposed by Max Weber in 1910 and used during World War II to analyse propaganda (Krippendorff, 2013), is recognized as a research technique in the social sciences for making replicable and valid inferences that can inform about patterns, trends, and changes in textual information. These kinds of studies play an important role not only in examining the past but also in possibly influencing the future for a field of study.

Recent examples of content analysis applied to professional journals include: an examination of two teacher education journals (Rock, Cheek, Sullivan, Jones, Holden \& Kang, 2016), one looking at types and trends of research design and statistical analysis in the Journal of Marital and Family Therapy (Parker, Chang, \& Thomas, 2016), and one in the Journal of Black Psychology (Cokley, Awosogba, \& Taylor, 2014).

Content analyses of journals can have a specific focus as in the Shapiro \& Pitts (2014) examination of disability sport in sport management literature or as in the coverage of men's issues in counseling in the Journal of Counseling \& Development and Counselor Education and Supervision (Evans, 2013) or the Rose, Friedman, Marquez \& Fernandez (2013) study that compared the English and Spanish versions of the American Association of Retired Persons' magazine for content on formal exercise and physical activity to reduce the risk of Alzheimer's disease.

Content analysis can also be more general without an explicit emphasis as in these studies: "A Content Analysis of Evaluation and the Health Professions Over 32 Years" (Sussman, 2010), "Moral education trends over 40 years: A Content Analysis of the Journal of Moral Education"(Lee \& Taylor, 2013), or the content analysis of the Journal of Career Assessment conducted by Whiston, Rose, Peterson \& Nguyen (2013).

Because the Journal of Aging and Health is a major journal examining the areas of health policy, services, and gerontology, it is important to explore for its readership any trends or patterns in the 
articles being published. The purpose of this study is to inform the readers of the Journal of Aging and Health about the published articles in the journal and to discover topic areas, trends, and perhaps under-represented areas that might be revealed by a content analysis of keywords associated with the journal articles during the study period. This paper complements the recent editorial by Markides, Downer, Schroeder \& Newby, 2016).

\section{Methodology}

Searches were conducted in Ageline, Medline, PsycINFO, and Web of Science for the term "content analysis" in the title along with "aging" in a second title search box and also "content analysis" was searched along with the journal title.

Accordingly, in September of 2015, the first author began reviewing and collecting the table of contents from JAHfor the ten years 2005-2014 and entered the article title information, journal volume and issue along with the keywords associated with each article into an Excel database.Only major articles were included; editorials, viewpoints, and letters were excluded.

The authors first listed each keyword alphabetically. Some keywords were almost identical such as "African American" and "African Americans," "body mass index" and "body mass index (BMI). Keywords were also grouped together when they were parallel or close approximations of the other such as 'dental caries' and 'oral health' and "heart disease" and "cardiac disease."Reviewing the master list multiple times allowed the authors to develop topical categories derived from the data. For example, the keywords "mental health," "depression," and "stress" were merged into a single category. All total, 23 categories of keywords were created.

\section{Results}

Numbers of articles and keywords. During this ten year period there were 584 articles, 75 issues, and 2572 keywords. The year with the most articles (84) and keywords (393) was 2013 when the Journal published a special issue on The ACTIVE Study. There was a trend evident in the number of articles published per year. The number of articles rose from 38 in the year 2005 to 68 in 2014. The total number of keywords associated with volumes 17 through 26 was 2572 and 1324 different keywords were found. The number of keywords also rose from 151 in the year 2005 to 290 in the year 2014. See Table 1.

Table 1 Numbers of Keywords, Articles and Issues, 2005-2014

\begin{tabular}{ccccccc}
\hline \multirow{2}{*}{ Year } & \multicolumn{2}{c}{ Keywords } & \multicolumn{2}{c}{ Articles } & \multicolumn{2}{c}{ Issues } \\
\cline { 2 - 7 } & Frequency & Percentage & Frequency & Percentage & Frequency & Percentage \\
\hline 2005 & 151 & 5.87 & 38 & 6.50 & 6 & 8.00 \\
2006 & 190 & 7.39 & 42 & 7.19 & 6 & 8.00 \\
2007 & 226 & 8.79 & 55 & 9.41 & 6 & 8.00 \\
2008 & 222 & 8.63 & 50 & 8.56 & 8 & 10.67 \\
2009 & 260 & 10.11 & 57 & 9.76 & 8 & 10.67 \\
2010 & 261 & 10.15 & 60 & 10.27 & 8 & 10.67 \\
2011 & 280 & 10.89 & 63 & 10.79 & 8 & 10.67 \\
2012 & 299 & 11.63 & 67 & 11.47 & 8 & 10.67 \\
2013 & 393 & 15.28 & 84 & 14.38 & 9 & 12.00 \\
2014 & 290 & 11.28 & 68 & 11.64 & 8 & 10.67 \\
\hline Total & 2572 & 100.0 & 584 & 100.0 & 75 & 100.0 \\
\hline
\end{tabular}

The numbers of issues each year increased from six in 2005 to eight (2008 to 2014) except in 2013 when there were nine issues. The increased number of articles resulted in an expansion of the number of pages published in each volume. However, average page length in articles from 2005 to 
2009 (20.70) was not significantly different from the average page length from 2010 to 2014 (20.66). See Table 2.

Table 2 Numbers of Pages Annually and Per Article

\begin{tabular}{ccccc}
\hline Year & Pages Annually & $\begin{array}{c}\text { Numbers of } \\
\text { articles }\end{array}$ & \multicolumn{2}{c}{$\begin{array}{c}\text { Length per article } \\
\text { Mean }\end{array}$} \\
\hline 2005 & 865 & 38 & 22.76 & \\
2006 & 891 & 42 & 21.21 & $(20.70)$ \\
2007 & 1044 & 55 & 18.98 & \\
2008 & 1011 & 50 & 20.22 & \\
2009 & 1199 & 57 & 21.04 & \\
\hline 2010 & 1255 & 60 & 20.92 & \\
2011 & 1399 & 63 & 22.21 & \\
2012 & 1485 & 67 & 22.16 & $(20.66)$ \\
2013 & 1513 & 84 & 18.01 & \\
2014 & 1414 & 68 & 20.79 & \\
\hline Total & 12076 & 584 & 20.68 & - \\
\hline
\end{tabular}

Categorization of Keyword. Keywords were assigned to categories based on a "best fit" strategy. While it is not possible to detail where each of the uniquekeywords were placed, a brief description is provided below of the major groupings of keywords that formed each of the 23 categories used to sort the different keywords.

Abuse: In this small category, the vast majority of the 39 keywords (79.5\%) dealt with substance abuse. Terms related to child abuse appeared four times and those relating to elder abuse appeared four times.

Activities: 62 of 87 keywords (71.3\%) involved "ADL," "activities" or "activity" as in activities of daily living or physical activity.

Aged/geriatric: "Aged" and "Aging" constituted 37.5\% of this large category of 269 keywords; "Old" and "Older" singularly and combined with other keywords represented another 34.9\%. The third largest group of keywords involved "Elderly" or "Elder" (16.7\%). The keyword "Retirement" appeared only 4 times.

Caregiver: This term or "Caregiving" constituted30of the 31 terms in this small category (about $1.2 \%$ of all the total keywords).

Cognitive: Approximately half of the keywords (45 of 92) dealt with cognition, cognitive impairment, or cognitive functioning. Another 21 (22.8\%) involved the term dementia. "Memory" was mentioned nine times and "Alzheimer's"seven times.

Country/Place: When the journal article did not involve the United States or its population, "China" appeared more than any other country $(n=7)$ followed by Mexico $(n=6)$. However, "Chinese" was counted 14 times and the city of Hong Kong appeared twice as did Taiwan and Singapore. "Latin America" appeared 8 times. Just those terms accounted for $42.3 \%$ of the 97 keywords in the country/place category.

Culture: Eight of the 10 keywords in the category contained "Cultural" or "Acculturation."

Death: "Mortality" constituted 55.3\% of the 47 keywords in this category. "End of Life" appeared 8 times (17.0\%).

Demographic: "Race" and "ethnicity" as general terms made up14.8\% of the 142 keyword category total. "Gender" constituted 14.1\%, "Socioeconomic" added $8.5 \%$ and "Rural" appeared nine times $(6.3 \%)$.

Falls: The keyword "Falls" or "Falling" occurred 28 times $(75.7 \%)$ in the 37 keyword category.

Health: The keyword "Health" appeared singularly or in combination with other terms (e.g.,health behavior, health promotion, health outcomes, self-rated health, healthy aging) 145 times and constituted $81.0 \%$ of the 179 keywords in this category. The next largest grouping was "Well-being" with a frequency of 13 ( $7.3 \%$ of the category). 
Inequality:"Disparities" as in health disparitiesand racial disparities was counted 19 times (48.7\%) in the 39 keyword category. Small frequencies were found for "Economic hardship" (twice), "Equity" (once), and "Health inequality" (twice)

Mental Health: The keyword "Depression" or "Depressive" (as in depressive symptoms) occurred 50 times, stress 17 times, and loneliness 10 times. These 3 keywords were responsible for two-thirds of the 114 keywords in the category. "Mental health" was counted ten times. "Psychotic" did not appear but "Antipsychotic medications" emerged once.

Physical Disability:The keyword "Disability" appeared singularly or with other keywords 60 times and represented about one-third of the terms in this 190 keyword category. "Function" and "Functional" as in functional limitations appeared 41 times, "Vision Impairment" or "Vision Loss" 15 times, "Exercise" 14 times, "Frail" or "Frailty" 11 times and "Hearing" 12times. "Hip Fracture" occurred only four times. These keywords captured $82.6 \%$ of all those in this category.

Residence: The most frequent keyword in this 87 keyword category was "Nursing" and it was most frequently paired with "Home" or "Homes" $(n=26,29.9 \%)$. "Long-term care" appeared 15 times $(17.2 \%)$, the term "Community" as in "Community-dwelling seniors" 14 times $(16.0 \%)$, and "Assisted living" occurred eight times (9.2\%). "Residential care facilities" emergedthree times and "Residential aged care" once.

Services: This category of 97 keywords contained no large groupings. "Health services" occurred 10 times $(10.3 \%)$ and it was followed by the term "Advance" as in "Advance care planning" or "Advance directives," occurring seven times (7.2\%) as did "Medicare." "Case management" appeared twotimes. "Adult day services" was found once as was "Satisfaction with care" and "Social services."

Sex: The smallest of the 23 categories contained 8 keywords. Five of these keywords involved "Sex," "Sexual" or "Sexuality." The keywords "Condom Use," "Masturbation," and "Gay Men" each occurred one time.

Social Support: Among the 122 keywords in this category, "Social" appeared 56 times (45.9\% of the category) and 37 times in combination with keywords such as "Networks," "Relationships," and so forth. "Social Support" occurred 19 times (15.6\%). Variations of the term "Religion" occurred 11 times as did "Family" or "Families," and "Neighborhood" was found 12times (9.8\%). "Marriage" or "Marital" appeared four times. These terms constituted $77 \%$ of the category. "Friends" or "friendship" was counted three times; parent-child relationship emerged once. Note that "Marital Status" occurred three times in the Demographic category.

Specific Ethnicity: The keyword "African American" appeared 21 times and "Black" once for $34.9 \%$ of the keywords in this category. "Hispanic" also occurred 21 times. Additionally, "Latinos" was counted four times and "Mexican American" six times. Korean Americans had the same frequency as "Latinos." "American Indian" appeared three times and "Asian American" once.

Specific Illness: Of the many diseases and conditions that may affect older adults, the largest category of 123 keywords ( $\mathrm{n}=25,20.3 \%$ ) involved "Cardiac," "Heart," or "Hypertension." There were 10 occurrences of "AIDS" or "HIV," the same number for "Cancer" or specific types of cancer, and also 10 occurrences of "Diabetes." Keywords associated with dental issues appeared nine times. "Arthritis" appeared in some form seven times. "Bone density" and "Osteoporosis" appeared three times each. "Parkinson's" and "Stroke" appeared two times each.

Study: Despite the size of the category (238keywords), it contained few large groupings. Specific studies (e.g., Berlin Aging Study) and theories (e.g.,"Ecological" =3) were mentioned a small number of times along instruments (e.g., "SF-36" =3). "Population-based study" had a frequency of eight and there were two additional "Population studies." "Longitudinal" was the largest keyword grouping with 20 occurrences; it was followed by "Quality of life" with 12 instances. "Epidemiology"and some variation of "Population" occurred 11 times. "Systematic review" had a frequency of four. "Qualitative" had a frequency of three, and "Content analysis," "Cost-benefit," and "Program Evaluation" only appeared once each. 
Weight: The keyword "Obesity" was counted 19 times and represented $44.2 \%$ of the 43 keyword category. It was followed by "Body mass index" or "BMI" with 13 occurrences (30.2\%). "Weight" occurred seven times $(16.3 \%)$.

Other: This category was composed of numerous $(n=418)$ keywords and most occurred only a few times each. The largest group was "Migration" with a frequency of $15(3.6 \%)$. "Education" or "Educational" appeared 12times for $2.9 \%$ of the category. "Smoking" and "Lifestyle" each appeared six times. "Volunteer" was counted 5 times and "Transportation" occurred four times. "Technology" was found three times.

The distribution of keyword categories is shown in Table 3. In keeping with a journal whose mission is to publish research on gerontology and health, it is not surprising that,excluding the Other category ( $16.3 \%$ of the total keywords), the two largest categories were for Aging $(n=269,10.5 \%$ of the total keywords) and Study ( $\mathrm{n}=238,9.3 \%$ of the total keywords). Other large categories were Physical Disability, and Health. The topics of Death, Weight, Abuse, Inequality, Falls, Caregiver, Culture, and Sex were the smallest categories and each contained fewer than 50 keywords.

Table 3 Frequencies of Keyword Categories by Years, 2005-2014

\begin{tabular}{lccccccccccc}
\hline & 2005 & 2006 & 2007 & 2008 & 2009 & 2010 & 2011 & 2012 & 2013 & 2014 & Total \\
\hline Abuse & 2 & 6 & 1 & 1 & 2 & 2 & 12 & 4 & 7 & 2 & 39 \\
Activities & 0 & 3 & 3 & 12 & 9 & 8 & 10 & 7 & 26 & 9 & 87 \\
Aged/ geriatric & 15 & 20 & 20 & 22 & 29 & 28 & 27 & 28 & 51 & 29 & 269 \\
Caregiver & 4 & 2 & 3 & 4 & 5 & 3 & 5 & 1 & 4 & 0 & 31 \\
Cognitive & 3 & 2 & 11 & 5 & 8 & 6 & 7 & 7 & 34 & 9 & 92 \\
Country/place & 1 & 15 & 2 & 10 & 9 & 10 & 7 & 10 & 12 & 21 & 97 \\
Culture & 1 & 0 & 0 & 1 & 1 & 1 & 3 & 1 & 0 & 2 & 10 \\
Death & 0 & 3 & 3 & 7 & 3 & 6 & 5 & 12 & 2 & 6 & 47 \\
Demographic & 8 & 9 & 12 & 12 & 8 & 18 & 16 & 19 & 22 & 18 & 142 \\
Falls & 0 & 1 & 4 & 2 & 9 & 1 & 4 & 3 & 7 & 6 & 37 \\
Health & 17 & 15 & 18 & 11 & 18 & 17 & 22 & 20 & 23 & 18 & 179 \\
Inequality & 2 & 2 & 2 & 6 & 6 & 6 & 2 & 9 & 2 & 2 & 39 \\
Mental health & 7 & 5 & 12 & 10 & 10 & 10 & 9 & 19 & 22 & 10 & 114 \\
Physical disability & 13 & 13 & 15 & 8 & 22 & 26 & 22 & 27 & 20 & 24 & 190 \\
Residence & 13 & 6 & 5 & 3 & 20 & 7 & 6 & 5 & 12 & 10 & 87 \\
Services & 9 & 12 & 13 & 10 & 11 & 9 & 9 & 7 & 13 & 4 & 97 \\
Sex & 0 & 0 & 3 & 0 & 1 & 1 & 0 & 1 & 2 & 0 & 8 \\
Social support & 1 & 9 & 12 & 15 & 13 & 6 & 19 & 13 & 12 & 22 & 122 \\
Specific Ethnicity & 4 & 5 & 5 & 4 & 8 & 5 & 16 & 6 & 5 & 5 & 63 \\
Specific illness & 12 & 13 & 16 & 11 & 7 & 16 & 10 & 11 & 13 & 14 & 123 \\
Study & 11 & 17 & 14 & 27 & 19 & 18 & 24 & 37 & 36 & 35 & 238 \\
Weight & 1 & 1 & 3 & 3 & 4 & 8 & 9 & 7 & 6 & 1 & 43 \\
Other & 27 & 31 & 49 & 38 & 38 & 49 & 36 & 45 & 62 & 43 & 418 \\
\hline Total & 151 & 190 & 226 & 222 & 260 & 261 & 280 & 299 & 393 & 290 & 2572 \\
\hline
\end{tabular}

Trend in category of keyword. Percent of change is a useful metric for describing how values have changed over time (Bennett \& Briggs, 2014). Accordingly, we calculated percent of change to compare changes in categories of keywords between two five-year time spans (2005-2009 and 2010-2014).

Table 4 displays percent change data for each category of keywords. Although many of the categories contain small frequencies, overall 12 categories appear to gain in popularity. The keyword categories of Weight, Culture, Abuse, Activities, and Cognitive increased the most (by 77.9\%, 60.7\% and $55.0 \%$ respectively). Eleven keywords categories decreased in a major way. These were the categories of Caregiver, Services, Residence, Sex, and Specific illness (decreasing by 50.3\%, 47.4\%, $41.4 \%, 31.1 \%$, and $25.3 \%$ respectively). 
Table 4 Percent of Change in Keywords

\begin{tabular}{lccccc}
\hline \multirow{2}{*}{ Categories } & \multicolumn{2}{c}{$2005-2006$} & \multicolumn{2}{c}{$2007-2008$} & Change \\
\cline { 2 - 5 } & $\mathrm{n}$ & $\%$ & $\mathrm{n}$ & $\%$ & $(\%)$ \\
\hline Abuse & 12 & $1.1 \%$ & 27 & $1.8 \%$ & $55.0 \%$ \\
Activities & 27 & $2.6 \%$ & 60 & $3.9 \%$ & $53.1 \%$ \\
Aged/ geriatric & 106 & $10.1 \%$ & 163 & $10.7 \%$ & $5.9 \%$ \\
Caregiver & 18 & $1.7 \%$ & 13 & $0.9 \%$ & $-50.3 \%$ \\
Cognitive & 29 & $2.8 \%$ & 63 & $4.1 \%$ & $49.6 \%$ \\
Country/place & 37 & $3.5 \%$ & 60 & $3.9 \%$ & $11.7 \%$ \\
Culture & 3 & $0.3 \%$ & 7 & $0.5 \%$ & $60.7 \%$ \\
Death & 16 & $1.5 \%$ & 31 & $2.0 \%$ & $33.4 \%$ \\
Demographic & 49 & $4.7 \%$ & 93 & $6.1 \%$ & $30.7 \%$ \\
Falls & 16 & $1.5 \%$ & 21 & $1.4 \%$ & $-9.6 \%$ \\
Health & 79 & $7.5 \%$ & 100 & $6.6 \%$ & $-12.8 \%$ \\
Inequality & 18 & $1.7 \%$ & 21 & $1.4 \%$ & $-19.6 \%$ \\
Mental health & 44 & $4.2 \%$ & 70 & $4.6 \%$ & $9.6 \%$ \\
Physical disability & 71 & $6.8 \%$ & 119 & $7.8 \%$ & $15.4 \%$ \\
Residence & 47 & $4.5 \%$ & 40 & $2.6 \%$ & $-41.4 \%$ \\
Services & 55 & $5.2 \%$ & 42 & $2.8 \%$ & $-47.4 \%$ \\
Sex & 4 & $0.4 \%$ & 4 & $0.3 \%$ & $-31.1 \%$ \\
Social support & 50 & $4.8 \%$ & 72 & $4.7 \%$ & $-0.8 \%$ \\
Specific Ethnicity & 26 & $2.5 \%$ & 37 & $2.4 \%$ & $-2.0 \%$ \\
Specific illness & 59 & $5.6 \%$ & 64 & $4.2 \%$ & $-25.3 \%$ \\
Study & 88 & $8.4 \%$ & 150 & $9.8 \%$ & $17.4 \%$ \\
Weight & 12 & $1.1 \%$ & 31 & $2.0 \%$ & $77.9 \%$ \\
Other & 183 & $17.4 \%$ & 235 & $15.4 \%$ & $-11.6 \%$ \\
\hline
\end{tabular}

\section{Discussion.}

Several observations can be made from this examination of keywords appearing in the Journal. First, while the general public associates aging with infirmity, disability, hearing loss, cognitive decline, and other negative terms, there is so much more to the study of aging and health. However, the total of the keyword categories representing physical and mental disability (the categories of Cognitive, Mental Health, and Physical Disability) sums to approximately 400 keywords or about $15 \%$ of all the keywords identified over the ten year study period.

Admittedly, the categories developed and placement of keywords within them might have been improved if an expert panel had been employed. This constitutes a limitation of the study. For instance, an argument could be made that many of the keywords in the Demographic category could have been merged into the Study Category and if that had happened, it would have reduced the overall number of categories and created a slightly larger one representing about $15 \%$ of the total keywords.

In those studies that identified or focused on specific ethnicities, the groupings of African American and Hispanics dominated.However, Native American, Alaska Native, and other minority groups such as Asian American received very little attention. Similarly,the category Inequality that aggregated racial and health disparities as keywords constituted a very small proportion of all the total keywords (less than 2\%). Discrimination as a keyword also appeared only once. "Blacks" as a keyword appeared only once - suggesting that term is fading from use. The LGBT community also received virtually no attention as there was only instance of a related keyword ("Gay Men") during the study period.

Although the World War II vets have just about all died off and most of the veterans from our involvement in Iraq and Afghanistan are far from retirement age, only three instances of the keyword "Veterans" seems to be a small number for a 10 year period. One would assume that as the 
development of assistive technology and adaptive equipment for our injured military have become available to the general public that more studies might have been conducted utilizing these keywords. Whether or not that assumption is correct, only one use of "assistive technology" suggests that the portion of the aging population who uses or could benefit from this technology may have been overlooked. "Technology" as a separate keyword, which could cover a whole range of settings, appeared only three times. Along that line, "Stroke" and "Parkinson's" occurred as keywords only twice each. "Wheelchairs" appeared once. "Medical decision making" emerged three times.

Although the number of keywords associated with the category of Mental Health was reasonably large $(n=114)$ and "Depression" with a frequency of 50 seems adequate, it is interesting that the keywords "Suicide" and "Suicidal Ideation" appeared only once each. "Elder mistreatment" or abuse occurred only four times. "Coping" appeared five times. Other terms that appeared infrequently were "Leisure" (three times) and "retirement" (four times). "Meaning in life" occurred twice.

Keywords that the authors expected would appear more than once were "Hospice," "Program evaluation," and "Cost-benefit." While it may be difficult to conduct studies with terminally ill patients, there would seem to be opportunities to use secondary data routinely collected by hospice staff in providing care to this special population. In this era when just about everything is computerized and information abundant, one might have wagered that more program evaluations and studies examining the cost-benefit of programs would have been conducted.

\section{Conclusion}

Attempting to classify unique keywords into logical and intuitive groupings is a challenging exercise that resulted in negotiation, discussion, and sometimes reclassification. The boundaries of the smaller categories allowed them to be better defined. However, having too many small categories creates greater difficulty in attempting to present the findings to the reader. We faced the perennial problem of deciding how many categories is too many and how few are too few. In spite of that struggle, this retrospective content analysis suggests that we may have identified a few areas that would benefit investigators who do not wish to conduct research in areas already crowded with a large number of studies.

\section{References}

[1] Krippendorff, K, Content analysis: An introduction to its methodology,3rd ed. ,Thousand Oaks, CA: Sage, 2013.

[2] Rock, M. L., Cheek, A. E., Sullivan, M. E., Jones, J. L., Holden, K. B., \& Kang J., Looking Back and Moving Ahead A Content Analysis of Two Teacher Education Journals. Teacher Education and Special Education: The Journal of the Teacher Education Division of the Council for Exceptional Children, 39(2016), 7-27.

[3] Parker, E.O., Chang, J. \& Thomas, V., A content analysis of quantitative research in the Journal of Marital and Family Therapy: A 10-year review. Journal of Marital and Family Therapy, 42 (2016) 3-18.

[4] Cokley, K., Awosogba, O., \& Taylor, D., A 12-year content analysis of the Journal of Black Psychology (2000-2011): Implications for the field of black psychology. Journal of Black Psychology, 40 (2014)215-238.

[5] Shapiro, D. \& Pitts, B. G., What little do we know: Content analysis of disability sport in sport management literature. Journal of Sport Management, 28 (2014) 657-671.

[6] Evans, M. P., Men in counseling: A content analysis of the Journal of Counseling \& Development and Counselor Education and Supervision, 1981-2011). Journal of Counseling and Development, 91(2013) SI 467-474. 
[7] Rose I. D., Friedman D. B., Marquez D. X., and Fernandez K., What Are Older Latinos Told About Physical Activity and Cognition? A Content Analysis of a Top-Circulating Magazine. Journal of Aging and Health 25 (2013)1143-1158.

[8] Sussman, S., A content analysis of Evaluation and the Health Professions over 32 years. Evaluation and the Health Professions, 33 (2010), 7-11.

[9] Lee, C-M. \& Taylor, M. J., Moral education trends over 40 years: A content analysis of the Journal of Moral Education (1971-2011). Journal of Moral Education, 42(2013) 399-429.

[10] Whiston, S. C., Rose, C., Peterson, J. M. et al., Content analysis of the Journal of Career Assessment. Journal of Career Assessment, 21 (2013) 367-377.

[11] Markides, K. S., Downer, B., Schroeder, \& Newby, T., Trends in JAH submissions and publications: 1989-2015. Journal of Aging and Health, 28(2016) 755-757.

[12]Bennett, J., \& Briggs, W.Using and understanding mathematics: A quantitative reasoning approach, 6th ed. , Boston, MA: Pearson,2014. 